\title{
Red cell distribution width is associated with acute myocardial infarction in young patients
}

\author{
Onur Kadir Uysal ${ }^{1}$, Mustafa Duran ${ }^{1}$, Bugra Ozkan², Durmus Yildiray Sahin ${ }^{3}$, \\ Kamuran Tekin ${ }^{3}$, Zafer Elbasan ${ }^{3}$, Fatih Akin ${ }^{1}$, Mehmet Balli ${ }^{3}$, Ozgur Gunebakmaz ${ }^{4}$, \\ Huseyin Arinc ${ }^{1}$, Mehmet Gungor Kaya ${ }^{5}$, Murat Cayli ${ }^{3}$ \\ ${ }^{1}$ Department of Cardiology, Kayseri Education and Research Hospital, Kayseri, Turkey \\ ${ }^{2}$ Department of Cardiology, Burdur Bucak State Hospital, Burdur, Turkey \\ ${ }^{3}$ Department of Cardiology, Adana Numune Education and Research Hospital, Adana, Turkey \\ ${ }^{4}$ Department of Cardiology, Kastamonu City Hospital, Kastamonu, Turkey \\ ${ }^{5}$ Department of Cardiology, Erciyes University School of Medicine, Kayseri, Turkey
}

\begin{abstract}
Background: There are few studies about predictors of ST elevation myocardial infarction (STEMI) in young patients. High red cell distribution width (RDW) levels were associated with adverse outcomes in patients with STEMI. We aimed to investigate the relationship between RDW and STEMI in young patients.
\end{abstract}

Methods: This study included 370 patients who presented to our hospital with acute myocardial infarction (Group 1: 198 young patients, < 45 ages for male, <55 ages for female, Group 2: 172 elderly patients) and 156 adults with normal coronary angiography as a control group (Group 3: 91 young patients, < 45 ages for male, < 55 ages for female, Group 4: 65 elderly patients).

Results: Compared with Group 3, Group 1 had a significantly higher value of RDW (Group 1 $R D W 14.1 \pm 1.1 \%$, Group $3 R D W 13.4 \pm 0.9, p 1<0.01)$. Value of $R D W$ was similar both of Group 2 and 4 (Group 2 RDW $13.7 \pm 1.2$, Group $4 R D W 13.5 \pm 0.9$, p2 = 0.1). After multivariate analysis, high levels of RDW were independent predictors of STEMI in young patients (OR: 0.337, $p<0.01$ ) together with gender (OR: 3.725, $p<0.01$ ), history of hyperlipidemia (OR: 25.172, $p<0.01)$ and high density lipoprotein cholesterol (OR: 1.088, $p<0.01$ ).

Conclusions: High levels of RDW were associated with STEMI in young patients. We think that $R D W$ is a widely available marker with no additional costs, in contrast to other novel markers of cardiovascular risk. (Cardiol J 2012; 19, 6: 597-602)

Key words: red cell distribution width, acute myocardial infarcion

\section{Introduction}

Red cell distribution width (RDW) is a laboratory measure of the variability in erythrocyte volume and is a readily available component of the rou- tine complete blood count. Recent studies have showed that high RDW levels were associated with adverse outcomes in patients with ST elevation myocardial infarction (STEMI) and heart failure [1,2]. High levels of RDW also associated with increased

Address for correspondence: Onur Kadir Uysal, MD, Kayseri Education and Research Hospital, Department of Cardiology, Hastaneler Caddesi, No: 78, Kocasinan, Kayseri, Turkey, tel: 00903523368884, e-mail: onurkadir@gmail.com 
mortality in patients with coronary artery disease (CAD) and patients undergoing percutaneous coronary intervention [3, 4]. A relation between RDW and complexity of CAD were shown in a previous study [5]. In spite of these associations, the mechanisms underlying elevated RDW are unclear in these patient groups. Inflammation and neurohumoral mediators may stimulate changes in red cell membrane, leading to increased RDW [6].

Investigators showed that only $5 \%$ of STEMI patients were younger than 40 ages [7]. Therefore, there are few studies about predictors of STEMI in young patients and no study has investigated the relationship between RDW and STEMI in this group. In this study, we aimed to investigate the relationship between RDW and STEMI in young patients.

\section{Methods}

\section{Patients}

We investigated 370 consecutive patients hospitalized with STEMI patients who admitted within $6 \mathrm{~h}$ from symptom onset and underwent primary percutaneous coronary intervention and 156 adults with normal coronary angiography as control groups. Patients were devided into 4 groups: Group 1 included $198(37 \%)$ young patients $(<45$ ages for males, $<55$ ages for females), Group $2-172(32 \%)$ elderly patients ( $>45$ ages for males, $>55$ ages for females) with STEMI, Group $3-91$ (17\%) young patients and Group $4-65$ elderly patients with normal coronary angiography.

Acute myocardial infarction was diagnosed at least 2 of the following: chest pain longer than 30 min which does not respond to nitrates; in 12-lead electrocardiography, a new $\mathrm{Q}$ wave longer than $40 \mathrm{~ms}$ and/or ST segment elevation $0.1 \mathrm{mV}$ on at least 2 extremity derivations or $0.2 \mathrm{mV}$ on precordial derivation; a 1.5 times, or higher, increase in serum creatine kinase-MB. Patients who had not any narrowing in coronary arteries were enrolled in the group 3 and 4 as normal coronary angiography.

Patients with a history of coronary intervention or coronary artery bypass, a history of cardiac failure, a history of renal disease (or serum creatinine level $>1.5 \mathrm{mg} / \mathrm{dL}$ ), anemia, clinical evidence of active infection, active cancer, hematological proliferative diseases, active or chronic inflammatory or autoimmune diseases, pregnancy, recent blood transfusion and patients with severe arrhythmia were excluded from the study.

Anemia was defined as hemoglobin levels lower than $13 \mathrm{~g} / \mathrm{dL}$ in men and $12 \mathrm{~g} / \mathrm{dL}$ in women, in accordance with the World Health Organization criteria [8].
The glomerular filtration rate (GFR) was evaluated by the simplified Modification of Diet in Renal Disease equation [9]:

$$
\text { GFR }=186 \times(\text { Serum Cr })^{-1.154} \times(\text { Age })^{-0.203}
$$

[ $\times 1.210$ if African-American] [ $\times 0.742$ if female].

Informed consent was obtained from all patients. The study was approved by our local ethical committee. All demographic and clinical data were collected prospectively.

\section{Laboratory analysis}

In all cases, blood samples were drawn at admission before starting any medication and were collected in tripotassium EDTA tubes. All measurements were performed $30 \mathrm{~min}$ after blood collection by an automatic blood counter (A Sysmex XE-2100, Symex, Kobe, Japan).

\section{Coronary angiography}

Quantitative coronary angiography was performed by two experienced interventional cardiologists who had no knowledge of the patients' clinical information by the Judkins technique via right femoral artery. Coronary arteries were imaged by utilizing right and left anterior oblique views with cranial and caudal positions. Injection of contrast medium (Iopromide, Ultravist-370; Schering AG, Berlin, Germany) was carried out by an automatic injector at a speed of 3-4 L/s for the left coronary artery and $2-3 \mathrm{~mL} / \mathrm{s}$ for the right coronary artery. Arteriographies were recorded at a speed of 25 frames/s.

\section{Statistical analysis}

All analyses were performed using SPSS V 17.0 for windows (version 17.0, SPSS, Chicago, Illinois, USA). Quantitative variables were expressed as mean value \pm SD for parametric variables and median and minimum-maximum levels for non-parametric variables. Comparison of parametric values between 2 groups was performed by means of Independent-Samples T test. Comparison of non-parametric values between 2 groups was performed by Mann-Whitney U test. Categorical variables were compared by the $\chi^{2}$ test. The Pearson test was used for correlation of parametric variables and the Spearman test was used test for non-parametric variables (p1: comparation between Group 1 and Group 3, p2: comparation between Group 2 and Group 4). Multivariate logistic regression analysis was performed to assess the effects of RDW and other variables on STEMI in young patients. A two-tailed $\mathrm{p}<0.05$ was considered significant. 
Table 1. Association of atherosclerotic characteristics of patients and red cell distribution width.

\begin{tabular}{|c|c|c|c|c|c|c|}
\hline & $\begin{array}{c}\text { Group } 1 \\
(n=198)\end{array}$ & $\begin{array}{c}\text { Group } 3 \\
(\mathbf{n}=91)\end{array}$ & P1 & $\begin{array}{l}\text { Group } 2 \\
(n=172)\end{array}$ & $\begin{array}{l}\text { Group } 4 \\
(n=65)\end{array}$ & P2 \\
\hline Age [years] & $41 \pm 4$ & $43 \pm 6$ & 0.01 & $64 \pm 10$ & $59 \pm 7$ & $<0.01$ \\
\hline Gender (male) & $154(77 \%)$ & $42(46 \%)$ & $<0.01$ & $107(62 \%)$ & $37(56 \%)$ & 0.4 \\
\hline Smoking & $82(41 \%)$ & $35(38 \%)$ & 0.5 & $71(41 \%)$ & $26(40 \%)$ & 0.8 \\
\hline Diabetes & $40(20 \%)$ & $12(13 \%)$ & 0.1 & $45(26 \%)$ & $12(23 \%)$ & 0.2 \\
\hline Hypertension & $42(21 \%)$ & $14(15 \%)$ & 0.2 & $66(38 \%)$ & $21(32 \%)$ & 0.4 \\
\hline Hyperlipidemia & $44(22 \%)$ & $8(8 \%)$ & $<0.01$ & $50(29 \%)$ & $22(33 \%)$ & 0.5 \\
\hline Family history of CAD & $66(33 \%)$ & $16(17 \%)$ & $<0.01$ & $42(24 \%)$ & $11(16 \%)$ & 0.3 \\
\hline Platelet count $\left(\times 10^{6}\right)$ & $238(117-463)$ & $249(133-456)$ & 0.04 & $235(109-474)$ & $245(110-436)$ & 0.5 \\
\hline Hemoglobin [mg/dL] & $14.5 \pm 1.4$ & $14.2 \pm 1.5$ & 0.2 & $14.3 \pm 1.2$ & $14.2 \pm 1.4$ & 0.5 \\
\hline RDW [\%] & $14.1 \pm 1.1$ & $13.4 \pm 0.9$ & $<0.01$ & $13.7 \pm 1.2$ & $13.5 \pm 0.9$ & 0.1 \\
\hline BUN [mg/dL] & $24(10-96)$ & $25(14-54)$ & 0.01 & $27(10-92)$ & $28(19-58)$ & 0.2 \\
\hline Creatinine $[\mathrm{mg} / \mathrm{dL}]$ & $0.89 \pm 0.2$ & $0.80 \pm 0.1$ & $<0.01$ & $0.93 \pm 0.2$ & $0.82 \pm 0.2$ & $<0.01$ \\
\hline Total cholesterol [mg/dL] & $185 \pm 44$ & $178 \pm 39$ & 0.2 & $178 \pm 36$ & $164 \pm 40$ & $<0.01$ \\
\hline LDL-C [mg/dL] & $125 \pm 39$ & $114 \pm 36$ & 0.04 & $119 \pm 32$ & $119 \pm 34$ & 0.9 \\
\hline $\mathrm{HDL}-\mathrm{C}[\mathrm{mg} / \mathrm{dL}]$ & $38 \pm 8$ & $47 \pm 12$ & $<0.01$ & $38 \pm 8$ & $43 \pm 13$ & 0.07 \\
\hline Triglycerides [mg/dL] & $135(40-467)$ & $132(34-357)$ & 0.8 & $130(32-460)$ & $125(50-424)$ & 0.8 \\
\hline $\mathrm{GFR}\left[\mathrm{mL} / \mathrm{min} / 1.73 \mathrm{~m}^{2}\right]$ & $100 \pm 28$ & $98 \pm 20$ & 0.9 & $82 \pm 26$ & $93 \pm 18$ & $<0.01$ \\
\hline
\end{tabular}

CAD - coronary artery disease; RDW - red cell distribution width; BUN — blood urea nitrogen; LDL-C — low density lipoprotein cholesterol; HDL-C - high density lipoprotein cholesterol; GFR - glomerular filtration rate; p1 - comparation between Group 1 and Group 3; p2 - comparation between Group 2 and Group 4

\section{Results}

The baseline characteristics and laboratory data of the 526 included patients are presented in Table 1 . There was no significant difference in the presence of diabetes mellitus, hypertension, smoking and levels of hemoglobin, total cholesterol, triglycerides and GFR rate between Group 1 and 3. In Group 1, age was younger, number of male sex and levels of RDW were higher than in Group 3. Also in Group 1, history of hyperlipidemia, presence of family history of CAD, levels of creatinine and LDL-cholesterol were higher than in Group 3 and levels of platelet count, HDL-cholesterol were lower than in Group 3.

In Group 2, age, levels of creatinine and GFR were higher than in Group 4 and level of total cholesterol was lower than in Group 4. Other parameters were similar in Group 2 and 4.

Compared with 3, Group 1 had a significantly higher value of RDW (Group 1 RDW $14.1 \pm 1.1 \%$, Group 3 RDW $13.4 \pm 0.9, \mathrm{p} 1<0.01)$. But levels of RDW were similar in Group 2 and 4 (Group 2 RDW $13.7 \pm 1.7$, Group 4 RDW $13.5 \pm 0.9$, p2 = 0.1).

Variables found to be statistically significant in univariate analysis between Group 1 and 3 were entered into multivariate logistic regression analysis. After multivariate analysis, high levels of RDW were independent predictors of STEMI in young patients (OR: $0.337,95 \%$ CI $0.298-0.558, \mathrm{p}<0.01$ ) together with gender (OR: 3.725, 95\% 1.446-9.597, $\mathrm{p}<0.01$ ), history of hyperlipidemia (OR: 25.172, $4.922-128.743, \mathrm{p}<0.01$ ) and high density lipoprotein cholesterol (OR: 1.088, 95\% CI 1.045-1.132, $\mathrm{p}<0.01$ ) (Table 2).

\section{Discussion}

To our knowledge, this is the first study to evaluate the relationship between RDW and STEMI in young patients. Our observations suggest that elevated levels of RDW are associated with an increased risk of STEMI in young patients.

Non-atherosclerotic CAD risk factors are congenital coronary abnormalities, connective tissue disorders, coronary artery aneurysm or coronary dissection, myocardial bridging and illicit drug usage [10-12]. Vasospasm and hypercoagulapathy also play a role in the pathophysiology of athero-thrombosis in young patients with STEMI [13]. Homozygous FV Leiden, protein C deficiency, antiphospholipid antibody syndrome, antithrombin III deficiency; long-term anticoagulation should be considered for a patient who has suffered a non-atherosclerotic thromboembolic event [14].

Coronary atherosclerosis is the main cause of STEMI in young patients (80\% of cases) [15]. 
Table 2. Multivariate analysis of determinants of myocardial infarction in young patients.

\begin{tabular}{lcccccccc}
\hline & & & & & & & \multicolumn{2}{c}{$95 \%$ Cl for Exp(B) } \\
\cline { 5 - 9 } & B & SE & Wald & df & Sig. & Exp(B) & Lower & Upper \\
\hline RDW & -1.089 & 0.221 & 24.206 & 1 & 0.000 & 0.337 & 0.218 & 0.519 \\
Gender & 1.315 & 0.483 & 7.415 & 1 & 0.006 & 3.725 & 1.446 & 9.597 \\
History of HPL & 3.226 & 0.833 & 15.006 & 1 & 0.000 & 25.172 & 4.922 & 128.743 \\
Family history of CAD & 0.762 & 0.462 & 2.725 & 1 & 0.099 & 2.143 & 0.867 & 5.298 \\
Platelets & 0.003 & 0.003 & 1.290 & 1 & 0.256 & 1.003 & 0.998 & 1.008 \\
BUN & 0.014 & 0.020 & 0.519 & 1 & 0.471 & 1.014 & 0.976 & 1.055 \\
Creatinine & -1.473 & 0.998 & 2.181 & 1 & 0.140 & .229 & 0.032 & 1.619 \\
LDL-C & 0.000 & 0.005 & 0.025 & 1 & 0.873 & .999 & 0.990 & 1.009 \\
HDL-C & 0.084 & 0.021 & 16.775 & 1 & 0.000 & 1.088 & 1.045 & 1.132 \\
Age & 0.024 & 0.041 & 0.338 & 1 & 0.561 & 1.024 & 0.945 & 1.109 \\
Constant & 5.669 & 3.322 & 2.911 & 1 & 0.088 & 289.758 & & \\
\hline
\end{tabular}

$\mathrm{Cl}$ — confidence interval; SE — standard error; df — degrees of freedom; RDW — red cell distribution width; HPL — hyperlipidemia; CAD — coronary artery disease; BUN — blood urea nitrogen; LDL-C — low density lipoprotein cholesterol; HDL-C — high density lipoprotein cholesterol

Atherosclerotic process begins at birth and significant lesions in coronary arteries can be detected as early as age 25 or 30 years. In a postmortem study that included 760 young adults (average age of $30-34$ years), $20 \%$ of males and $8 \%$ of females were found to have atherosclerotic CAD [16]. All well-known classic risk factors for atherosclerosis are acceptable for young patients with STEMI. In addition to the classical risk factors there are new predictors for atherosclerosis in young patients such as lipoprotein a, fibrinogen and uric acid [17].

$\mathrm{RDW}$ is a marker of the variability in erythrocyte volume and is a routinely available component of the complete blood count. Elevated RDW reflects heterogeneity of cell sizes in the peripheral blood smear [18]. Elevated RDW levels can be seen in conditions of ineffective red cell production (such as iron deficiency, B12 or folat deficiency and hemoglobinopathies), increased red cell destruction (such as hemolysis) and with blood transfusion [19].

The underlying mechanism by which high levels of RDW are associated with STEMI is unclear. Inflammation have an important role in atherosclerotic process [20]. Inflammatory cytokines which are activated in STEMI have been found to suppress the erythrocyte maturation, so juvenile erythrocytes enter into circulation [21]. Also elevated levels of neurohumoral mediators stimulate erythropoiesis, for example, angiotensin II may affect the erythroid progenitor cells with a direct stimulation [6]. In addition, levels of erythropoietin increases independent of hemoglobin levels and adrenergic activation may act bone marrow response in patients with STEMI [22]. There is a substantial genetic contribution to red cell size in general population [23]. The variability in circulation red cell sizes may increase with these mechanisms. The investigators showed a relationship between generalized inflammation and elevated levels of RDW and demonstrated an association of RDW with high sensitive C-reactive protein and erythrocyte sedimentation rate which are indicators of inflammation [24].

Several studies evaluated a relation between increased RDW and morbidity and mortality of CAD. Investigators showed that an increased RDW was independently associated with in hospital and long-term all-cause mortality and coronary events in STEMI, non-ST elevation MI and unselected population of male patients referred for coronary angiography $[2,25,26]$. Also in other studies a high admission RDW was independently associated with worse reperfusion and increased risk in-hospital and long-term cardiovascular mortality in patients with STEMI undergoing primary percutaneous intervention [27]. In a study of 7556 adult participants (devided into 3 categories according to their 10 -year Framingham risk of hard CAD events; $<10 \%, 10$ $-20 \%,>20 \%$ ) were found that a higher RDW might be a powerful independent predictor of future coronary heart disease risk [28]. Isik et al. [5] demonstrated that RDW was independently associated with the presence of $\mathrm{CAD}$ and coronary complexity of CAD as assessed by the SYNTAX score.

In our study, we found that high levels of RDW were associated with STEMI in young patients. As we mentioned in the result section, levels of RDW were similar in elderly patients with STEMI and normal coronary angiography. Levels of RDW were 
higher only in young patients with STEMI in our study. It is well known that RDW is higher in elderly patients, also in patients with and without CAD. This situation may explain the similar value of RDW in both elderly groups.

The underlying mechanisms between RDW and cardiovascular morbidity and mortality have not yet been clearly demonstrated. Some hypotheses were suggested for this association. Atherosclerotic process was related with inflammatory reactions and elevated levels of RDW were associated with inflammation markers such as high sensitive C-reactive protein and erythrocyte sedimentation rate. As we mentioned above related inflammatory cytokines suppress the erythrocyte maturation, so juvenile erythrocytes enter into circulation. Also elevated levels of neurohumoral mediators and adrenergic activation stimulate erythropoiesis. In addition, there is a substantial genetic contribution to red cell size in general population [23]. The variability in circulation red cell sizes may increase with these mechanisms.

Only hemoglobin levels were measured in this study and other factors including, levels of iron, vitamin B12, folate and mean corpuscular volume were not measured. But also, we did not implicate the patients with anemia to our study. We believe that this makes it highly unlikely that the predictive value of RDW was related to iron deficiency. Furthermore, the incidence of clinically significant vitamin B12 and folate deficiency is low in a modern population. In a study of 15,852 adult participants were found that RDW was an independent predictor of mortality and the investigators concluded that the relationship between RDW and mortality was not confounded by anemia-related deficiencies such as vitamin B12, folate and iron [29].

History of hyperlipidemia was found to be more common in group 1 than group 3. Based on this result, the more common presence of history of hyperlipidemia in young patients indicates the importance of familial hypercholesterolemia in this group, as expected.

HDL levels were lower in Group 1 than Group 3. Several studies indicate significantly higher levels of triglicerydes and total cholesterol and lower concentration of HDL-cholesterol in young patients with CAD, compared with elderly patients [30]. In our study, LDL-cholesterol were higher, HDL-cholesterol was lower in young patients with STEMI than normal group. But we did not find any significant difference between elderly groups. We did not have any information about patient's medication.
This result may related with usage of antihyperlipidemic drugs by elderly patients.

\section{Limitations of the study}

There are several limitations in our study. We did not evaluate other novel risk factors for STEMI in young patients such as fibrinogen, CD 40 ligand, FV Leiden, protein $\mathrm{C}$ and antithrombin III and could not evaluate the medical history of patients. Only hemoglobin levels were measured in this study and other factors including, levels of iron, ferritin, vita$\min$ B12 and folate were not measured. We did not evaluate etiology of STEMI in our study groups (vasospasm, congenital coronary abnormalities and others). So we could not compare levels of RDW according to atherosclerotic origin and nonatherosclerotic origin.

\section{Conclusions}

We found that high levels of RDW were associated with STEMI in young patients. Also we think that RDW is a widely available marker with no additional costs, in contrast to other novel markers of cardiovascular risk.

\section{Conflict of interest: none declared}

\section{References}

1. Felker GM, Allen LA, Pocock SJ et al. Red cell distribution width as a novel prognostic marker in heart failure: Data from the CHARM Program and the Duke Databank. J Am Coll Cardiol, 2007; 50: 40-47.

2. Uyarel H, Ergelen M, Cicek G et al. Red cell distribution width as a novel prognostic marker in patients undergoing primary angioplasty for acute myocardial infarction. Coron Artery Dis, 2011; 22: 138-144.

3. Anderson JL, Ronnow BS, Horne BD et al. Usefulness of a complete blood count derived risk score to predict incident mortality in patients with suspected cardiovascular disease. Am J Cardiol, 2007; 99: 169-174.

4. Poludasu S, Marmur JD, Weedon J et al. Red cell distribution width (RDW) as a predictor of long-term mortality in patients undergoing percutaneous coronary intervention. Thromb Haemost, 2009; 102: 581-587.

5. Isik T, Uyarel H, Tanboga IH et al. Relation of red cell distribution width with the presence, severity, and complexity of coronary artery disease. Coron Artery Dis, 2012; 23: 51-56.

6. Weiss G, Goodnough LT. Anemia of chronic disease. N Engl J Med, 2005; 352: 1011-1023.

7. Lamm G. The epidemiology of acute myocardial infarction in young age groups. In: Roskamm H ed. Myocardial infarction at young age. Springer-Verlag, Berlin 1981: 5-12.

8. World Health Organization. Nutritional Anemia: Report of a WHO Scientific Group World Health Organization. World Health Organization, Geneva 1968. 
9. Stevens LA, Coresh J, Greene T et al. Assessing kidney function measured and estimated glomerular filtration rate. $\mathrm{N}$ Engl J Med, 2006; 354: 2473-2483.

10. Harikrishnan S, Jacob SP, Tharakan J et al. Congenital coronary anomalies of origin and distribution in adults: A coronary angiographic study. Indian Heart J, 2002; 54: 271-275.

11. Rhew EY, Ramsey-Goldman R. Premature atherosclerotic disease in systemic lupus erythematosus: Role of inflammatory mechanisms. Autoimmun Rev, 2006; 5: 101-105.

12. Longheval G, Badot V, Cosyns B et al. Spontaneous coronary artery dissection: Favorable outcome illustrated by angiographic data. Clin Cardiol, 1999; 22: 374-375.

13. Hosoi T, Koyama $\mathrm{Y}$, Tange $\mathrm{S}$ et al. Acute myocardial infarction due to vasospasm in a 13 year old boy. Jpn Circ J, 1997; 61: 536-542.

14. Foy P, Moll S. Thrombophilia: 2009 update. Curr Treat Options Cardiovasc Med, 2009; 11: 114-128.

15. Choudhury L, Marsh J. Myocardial infarction in young patients. Am J Med, 1999; 107: 254-261.

16. McGill HC Jr, McMahan CA, Zieske AW et al. Association of coronary heart disease risk factors with microscopic qualities of coronary atherosclerosis in youth. Circulation, 2000; 102: 374-379.

17. Tatli E, Aktoz M, Buyuklu M et al. The relationship between coronary artery disease and uric acid levels in young patients with acute myocardial infarction. Cardiol J, 2008; 15: 21-25.

18. Marsh WL, Bishop JW, Darey TP. Evaluation of red cell volume distribution width (RDW). Haematol Pathol, 1987; 1: 117-123.

19. Förhécz Z, Gombos T, Borgulya $G$ et al. Red cell distribution width in heart failure: prediction of clinical events and relationship with markers of ineffective erythropoiesis, inflammation, renal function, and nutritional state. Am Heart J, 2009; 158: 659-666.

20. Libby P. What have we learned about the biology of atherosclerosis? The role of inflammation. Am J Cardiol, 2001; 88: 3J-6J.
21. Pierce CN, Larson DF. Inflammatory cytokine inhibition of erythropoiesis in patients implanted with a mechanical circulatory assist device. Perfusion, 2005; 20: 83-90.

22. Ferrario M, Massa M, Rosti $V$ et al. Early haemoglobin-independent increase of plasma erythropoietin levels in patients with acute myocardial infarction. Eur Heart J, 2007; 28: 1805-1813.

23. Lin JP, O'Donnell CJ, Jin L et al. Evidence for linkage of red blood cell size and count: genome-wide scans in the Framingham Heart Study. Am J Hematol, 2007; 82: 605-610.

24. Lippi G, Targher G, Montagnana $\mathrm{M}$ et al. Relation between red blood cell distribution width and inflammatory biomarkers in a large cohort of unselected outpatients. Arch Pathol Lab Med, 2009; 133: 628-632.

25. Azab B, Torbey E, Hatoum $\mathrm{H}$ et al. Usefulness of red cell distribution width in predicting all-cause long-term mortality after non-ST-elevation myocardial infarction. Cardiology, 2011; 119: 72-80.

26. Cavusoglu E, Chopra V, Gupta A et al. Relation between red blood cell distribution width (RDW) and all-cause mortality at two years in an unselected population referred for coronary angiography. Int J Cardiol, 2010; 141: 141-146.

27. Karabulut A, Uyarel H, Uzunlar B et al. Elevated red cell distribution width level predicts worse postinterventional thrombolysis in myocardial infarction flow reflecting abnormal reperfusion in acute myocardial infarction treated with a primary coronary intervention. Coron Artery Dis, 2012; 23: 68-72.

28. Zalawadiya SK, Veeranna V, Niraj A et al. Red cell distribution width and risk of coronary heart disease events. Am J Cardiol, 2010; 106: 988-993.

29. Perlstein TS, Weuve J, Pfeffer MA et al. Red blood cell distribution width and mortality risk in a community-based prospective cohort. Arch Intern Med, 2009; 169: 588-594.

30. Chen L, Chester M, Kasaki J. Clinical factors and angiographic features associated with premature coronary artery disease. Chest, 1995; 108: 364-369. 